
\title{
$\begin{array}{ll}\text { Research Square } & \begin{array}{l}\text { Preprints are preliminary reports that have not undergone peer review. } \\ \text { They should not be considered conclusive, used to inform clinical practice, } \\ \text { or referenced by the media as validated information. }\end{array}\end{array}$
}

\section{Diagnosis and Treatment for Squamous Cell Carcinoma of the Oral Tongue in a Patient with Fanconi Anemia}

\section{Siyao Deng}

School of Medicine, University of Electronic Science and Technology of China, Chengdu, China

\section{Wenjing Ye}

School of Medicine, University of Electronic Science and Technology of China, Chengdu, China

\section{Shichuan Zhang}

Department of radiation oncology, Sichuan Cancer Hospital \& Institute, Sichuan Cancer Center, School of Medicine, University of Electronic Science and Technology of China, Chengdu, China

\section{Peng Zhang}

Department of radiation oncology, Sichuan Cancer Hospital \& Institute, Sichuan Cancer Center, School of Medicine, University of Electronic Science and Technology of China, Chengdu, China

\section{Yanqiong Song}

Department of radiation oncology, Sichuan Cancer Hospital \& Institute, Sichuan Cancer Center, School of Medicine, University of Electronic Science and Technology of China, Chengdu, China Jie Zhou

Department of radiation oncology, Sichuan Cancer Hospital \& Institute, Sichuan Cancer Center, School of Medicine, University of Electronic Science and Technology of China, Chengdu, China Jinyi Lang

Department of radiation oncology, Sichuan Cancer Hospital \& Institute, Sichuan Cancer Center, School of Medicine, University of Electronic Science and Technology of China, Chengdu, China

\section{Shun Lu ( $\square$ lushun1982@live.cn )}

University of Electronic Science and Technology of China

\section{Research}

Keywords: Fanconi anemia, radiotherapy, cisplatin, head and neck squamous cell carcinoma, toxicity

Posted Date: November 19th, 2020

DOI: https://doi.org/10.21203/rs.3.rs-108060/v1

License: (c) (1) This work is licensed under a Creative Commons Attribution 4.0 International License. Read Full License 


\section{Abstract}

\section{Background}

Fanconi anemia (FA) is a rare genetic disorder characterized by congenital anomalies, progressive bone marrow failure and high susceptibility to various solid tumors, especially head and neck squamous cell carcinoma (HNSCC). Management of FA patients with head and neck cancer is a challenge due to increased risk of surgery, poor tolerance of chemotherapy, and severe myelotoxicity of radiotherapy.

\section{Methods}

We present a case of a 33-year-old man with carcinoma of the oral tongue (T1N2M0), who experienced prolonged and profound bone marrow failure as a consequence of concurrent cisplatin/radiation. The young patient who developed HNSCC without risk factors, the myelotoxicity after exposure to the platinum-based agent cisplatin and the further evaluation of phenotypic characteristics raised suspicion of FA. Whole exome sequencing performed for the patient and parents ultimately established the diagnosis of FA.

Results

Genetic testing in the 23 FANC genes revealed two novel heterozygous mutations, c.367C>T (p.Gln123*) and c.3971_3972delCGinsTT (p.Pro13241.cu) in FANCA gene of the patient, which were inherited from his father and mother, respectively. Radiotherapy with reduced dose has successfully alleviated the symptoms of tumor invasion and progression, and the radiation-related side effects were acceptable. Unfortunately, the patient died of locoregional disease progression.

\section{Conclusions}

This case highlights the importance of considering the diagnosis of FA in young patients who develop HNSCC in the absence of risk factors, thus permitting more effective oncological treatment strategies and improved outcomes. In general, any decision on different modalities of management in such patients should be based on a balance between locoregional control and therapeutic toxicity.

\section{Background}

Fanconi anemia (FA) is a multisystem disorder characterized by congenital abnormalities, progressive bone marrow failure and pancytopenia, high susceptibility to acute myeloid leukemia and solid tumors (1). In FA patients, hematological abnormalities usually appear within the first decade of life, which eventually develop pancytopenia and even bone marrow failure (2). Congenital anomalies include skeletal malformations (short stature, microcephaly, or hypoplastic thumb), organ abnormalities (renal, ophthalmic, ear, cardiac, genital), abnormal skin pigmentation like café au lait spots, and mental retardation (3). These further prove that FA is a pleiotropic disease on the phenotype. 
At least 23 genes have been implicated in FA that are responsible for the various complementation groups (A-C, D1, D2, E-G, I, J, L-W, Y). Mutations in FANCA are the most common, involved in $60-65 \%$ of reported cases (4). All mutations in FA genes are inherited in an autosomal recessive manner, with the exception of FANCB and RAD51, which are inherited in an X-linked manner and autosomal dominant manner, respectively (5). Products of these FA genes appear to interact in a common cellular pathway and participate in various aspects of DNA repair, particularly DNA interstrand crosslink repair, genomic stabilization, and regulation of downstream proteins $(6,7)$. Eight of the FA proteins (FANCA/-B/-C/-E/ $-F /-G /-L /-M)$ are constitutively assembled in a multisubunit enzyme core complex to mediate the monoubiquitination of FANCD2 and FANCl after DNA damage, which is a central step in the FA pathway, as a response to cross-linking agents or reactive oxygen species (8-10). Any mutation or loss of the FA core complex components will result in failure of monoubiquitination. In addition, there is interaction with BRCA1/BRCA2 complexes and an array of proteins involved in cell cycle checkpoint regulation and DNA damage repair $(11,12)$. Defects in any of these FA proteins result in genomic instability, defective DNA repair mechanisms and an increased risk of cancer (13).

Although the risk of hematological abnormalities is higher in childhood, solid tumors are more common in adults (14). Interestingly, FA patients have a significantly higher risk of head and neck cancer, and most of them occur in the oral cavity, in which tongue carcinomas are the most common subsite (1). Compared with the general population, the incidence of head and neck cancer in FA patients has increased by 500fold, with a cumulative incidence rate of $14 \%$ by the age of 40 years (15). FA patients with hematopoietic dysfunction usually require supportive treatment such as blood transfusions, hematopoietic growth factor and androgen replacement therapy. Bone marrow transplantation is the preferred treatment option, but is associated with a high risk of the late development of FA-related head and neck cancer (16-18). The management of FA patients with head and neck cancer requires multidisciplinary coordination and is extremely challenging. First, potential pancytopenia results in increased risk of surgery and poor tolerance to nonsurgical treatment. Second, defective DNA repair mechanism and chromosomal fragility increase the sensitivity to cytotoxic chemotherapeutic agents such as cisplatin, typically used in head and neck cancer (19). Third, defects in the identification and homologous recombination repair disorders of DNA damage may induce abnormal toxicity of radiotherapy (20).

Here, we reported a new case of a young man with FA treated at our institution for the tongue squamous cell carcinoma, reviewed his clinical course and described the treatment-related toxicity. Institutional approval was obtained and written consent was received from the patient's parents for this article.

\section{Methods}

\section{The patient and initial treatment}

A 33-year-old man without history of drinking and smoking was admitted to our hospital for examination of a lump on the right side of the tongue dorsum. Oral examination revealed a borderline ulcerative hyperplasia at the right side of tongue dorsum with white plaque covering it. No superficial lymph node 
enlargement was noted on neck examination. Computer tomography (CT) scan showed enhanced signals in the right tongue dorsal lesion and multiple bilateral cervical lymph nodes without any distant metastatic spread. Dynamic contrast-enhanced magnetic resonance imaging (DCE-MRI) of the maxillofacial region revealed a $1.6 \times 1.4 \times 1.7 \mathrm{~cm}$ mass located at the right side of tongue dorsum, with significant cervical lymphadenopathy (Figure 1A). A pathological biopsy performed under local anesthesia demonstrated highly differentiated squamous cell carcinoma (Figure 2A, 2B). As a result, a diagnosis of T2N2M0 (stage III) squamous cell carcinoma of the oral tongue was made.

Initial hematological parameters showed features of mild pancytopenia, which ruled out aggressive surgical interventions. After receiving supportive care until the blood count returned to normal, the patient was treated with concurrent cisplatin/radiation, the standard of care for locally HNSCC, according to the National Comprehensive Cancer Network (NCCN) guidelines. He received 13 days of single-dose cisplatin therapy $\left(40 \mathrm{mg} / \mathrm{m}^{2}, \mathrm{~d} 1-\mathrm{d} 3\right)$ combined with 11 fractions of daily intensity modulated radiation therapy (IMRT) (GTV 2.2Gy/f, GTVln 2.2Gy/f, CTV 2.0Gy/f, CTVln 1.8Gy/f, 5 fractions/week). However, the treatment discontinued due to the pancytopenia (total leukocyte count: $2.02 \times 10^{9} / \mathrm{L}$; hemoglobin count: $72 \mathrm{~g} / \mathrm{L}$; platelet count: $87 \times 10^{9} / \mathrm{L}$ ). Additionally, he continued to have profound and persistent myelosuppression despite blood product support and daily administration of subcutaneous recombinant granulocyte-colony-stimulating factor.

\section{Phenotypic features and family history}

To determine the cause of the patient's continued pancytopenia, he underwent a bone marrow aspiration and biopsy which revealed a severely hypoplastic marrow with no granuloma, hematopoiesis, nor malignant cells. Given the development of HNSCC at a young age in the absence of risk factors, the development of profound and persistent bone marrow failure induced by the low dose of cisplatin, the possibility of a genetic DNA repair defect was proposed. Through a comprehensive evaluation of phenotypic characteristics, several physiological characteristics of FA were determined. The patient was physically retarded including short stature and microcephaly. In addition, he generalized hyperpigmentation of the skin, accompanied by a few café au lait spots, particularly of the upper extremities (Figure 3A, 3B). The patient was born to non-close relative parents and had a younger brother of normal stature. Besides, his parents displayed no phenotypic signs of hereditary syndromes.

\section{Genetic testing}

In consideration of his abnormal morphological features and unexplained hypoplastic marrow, the patient was advised for molecular genetic testing. After obtaining the informed consent of the family, peripheral blood was collected from proband and the parents for whole exome sequencing. Apart from the mutation analysis of the patient, targeted mutational analysis was performed on the parents to determine if they also had the mutations identified in the proband. As a result, the molecular study revealed compound heterozygous mutations in the FANCA gene of the proband. One mutation was a previously unreported heterozygous nonsense mutation, c.367C >T (p.G $\left.\ln 123^{\star}\right)$, which caused the protein 
encoded by the FANCA gene to terminate prematurely at the 123rd amino acid. The other one was a novel heterozygous mutation, c.3971_3972delCGinsTT (p.Pro13241.cu), caused by a combination of c.3971 C>T (p.Pro13241.cu) and c.3972G>T (Pro1324Pro) linkage mutations (Figure 4A, 4B). The two heterozygous mutations were inherited from his father and mother, respectively (Figure 5). The variant was queried against the public version of the Human Gene Mutation Database (HGMD, http://www.hgmd.cf.ac.uk/). Finally, clinical diagnosis of FA was made on the basis of clinical features, bone marrow biopsy and genetic testing analysis.

\section{Results}

In consideration of the pancytopenia and substantially increased risk of toxicity with chemotherapy, especially DNA cross-linking agents like cisplatin, thus immediate discontinuation of cisplatin was determined. However, during the 8 months of bone marrow recovery therapy with cessation of concurrent cisplatin/radiation, DCE-MRI showed the tumor had increased in size to $3.7 \times 3.0 \times 3.1 \mathrm{~cm}$ (Figure 1B). Given his aggressive disease, the risk of locoregional failure and symptomatic progression was considered to outweigh the risk of treatment-related adverse event. After multidisciplinary diagnosis and treatment with experts of hematology, radiation oncology and oncology internal medicine, we modified the IMRT plan to the dose of GTV 2.0G $* 30 \mathrm{f}$, GTVInL 2.0Gy*30f, GTVInR 2.0G $* 30 \mathrm{f}, \mathrm{CTV} 1.8 \mathrm{~Gy} * 30 \mathrm{f}$ to reduce radiation toxicity. After improvement of blood cells with the intensive support care, the patient completed 21 fractions of IMRT and the tumor size had decreased to $3.2 \times 2.3 \times 2.8 \mathrm{~cm}$ (Figure 1C). During the treatment, he had Radiation Therapy Oncology Group (RTOG) grade 1 toxicity of skin and mucosa. Unfortunately, he deteriorated progressively after discharge and died from disease progression after 5 months of follow-up.

\section{Discussion}

FA is a disease in which underlying human genome instability plays an important role. With a rare incidence of one to five cases per million persons, heterozygous carrier frequency of FA is one in 300 persons (21). In certain populations, such as Ashkenazi Jews and Spanish gypsies, the carrier frequency is believed to be as high as $1 / 100(22,23)$. Cases of FA are usually diagnosed through recognition of congenital and hematological abnormalities, with a median age of diagnosis at 4.8-7.5 years $(24,25)$; therefore, it is rarely considered for diagnosis in adults.

FA patients are at high risk for the development of hematological malignancies and various solid tumors especially HNSCC. Onset of tumors in patients with FA is as early as 16 to 31 and shows a more aggressive behavior. Interestingly, $22 \%$ of FA patients who develop solid tumors are diagnosed with FA only after discovery of their cancer (26). In this case, the diagnosis of FA was atypical, presenting in an adult, and made only after unexpected toxicity of DNA cross-linking agents in an initially assumed sporadic malignancy. Therefore, FA should be considered in early-onset malignancies, especially if a typical FA-related malignancy is diagnosed. In addition, severe hematologic abnormalities of the patient did not appear until initiation of systemic tumor therapy. In some FA patients, genomic duplication is 
reversed to wild-type alleles in hematopoietic tissues, which can induce spontaneous correction of hematological alterations. In non-hematopoietic tissue, the biallelic inactivation stills remains, and thus the patient harbors mosaicism for the mutated FA gene (27). This phenomenon may explain a milder haematological phenotype for this patient with FA. Notably, FA is a heterogeneous disease and $30 \%$ of FA patients can present without any congenital abnormalities $(3,28)$. Thus, lack of physical manifestations does not preclude a diagnosis of FA.

Chemotherapy is one treatment option of most patients presenting with recurrent/metastatic HNSCC. It also can be used as a radiation sensitizer. Cisplatin is a DNA cross-linking agent recommended to be used in patients with oral cancer. However, people with FA have a substantially increased risk of toxicity to this kind of agent. It has been reported that one patient developed high-grade mucositis, cytopenia, tracheal stenosis, radiation pneumonitis, recurrent pneumonia, persistent myelosuppression and hemorrhage after receiving radiotherapy with cisplatin, bleomycin and methotrexate (29). In the present patient, the cisplatin chemotherapeutic regime was given before the diagnosis of FA. Severe and persistent bone marrow failure was observed and the treatment plan was interrupted, which most likely resulted from the chemotherapy. Although concurrent radio-chemotherapy is recommended to treat inoperable oral cancer patients, patients with FA may experience potentially fatal bone marrow toxicity from concurrent radio-chemotherapy; therefore, cytotoxic chemotherapy is discouraged for these patients. Most importantly, timely identification of underlying FA would lead to a different therapeutic approach with a potential better outcome.

However, FA patients with HNSCC tend to have a poor prognosis, which is associated with the aggressive disease presentation and the limitations of multimodality therapy due to potential bone marrow failure (30). Given the bone marrow abnormalities and low tolerance to standard therapies, treatment is more challenging in these patients. Radiotherapy has been served as a definitive treatment in many FA patients with HNSCC given the significant but acceptable toxicity; 70 Gy was recommended for definitive initial treatment (31). However, these patients have a significantly increased complication rate compared with the general HNSCC population, sometimes patients with FA even cannot complete full course of the treatment (32). Based on the International Fanconi Anemia Registry (IFAR) series, radiation-related side effects including anemia, thrombocytopenia, myelosuppression, skin ulceration and stenosis of the trachea were observed in the 8 FA patients treated with 40-61Gy radiotherapy (15). Previous studies have reported that doses of radiotherapy ranged from 3.2 to $80 \mathrm{~Gy}$ and the acute hematologic toxicity was individualized. Severe radiation-related toxicity could occur in a low dose such as $8 \mathrm{~Gy}$ in some FA patient, while the higher dose was acceptable in others (33-35). In this case, the patient was initially treated with 24.2 Gy IMRT in 11 fractions. After 8 months interruption of treatment, standard chemotherapy agent was not considered after diagnosis of FA in the patient due to the risk of unacceptable myelosuppression and irreversible aplastic anemia. IMRT of $42 \mathrm{~Gy} / 21 \mathrm{f}$ was then treated to the progressed tumor. From the DCE-MRI evaluation, radiotherapy has successfully alleviated the symptoms of tumor invasion and progression, and the radiation-related side effects were acceptable. Combined with the above evidence, a precise dose of radiotherapy with careful toxicity assessment is 
needed for each patient to enhance the treatment, since other therapeutic options are not available because of the high risk.

For FA patients with HNSCC, longer duration treatment courses with lower dose per fraction of radiotherapy may maximize the therapeutic effect and reduce complications. Furthermore, in view of the adaptability of dose and target volume, application of the technique allowing the best sparing of healthy tissues, such as IMRT with image-guided radiotherapy and intensity-modulated proton therapy, may be a safer option (36). Overall, any decision on different modalities of management in such patients should be based on a balance between locoregional control and therapeutic toxicity.

\section{Conclusions}

In general, the patient with a classically FA-associated early-onset cancer in this case can serve as a reminder to consider the diagnosis of FA in young patients who develop HNSCC in the absence of risk factors. Clinicians involved in the care of such patients should conduct an in-depth investigation of the patient's family history and accurate genetic testing, in order to timely diagnosis with FA to permit more effective oncological treatment strategies and improved outcomes. Moreover, for the treatment of inoperable FA patients with local HNSCC, chemotherapy is not suggested due to the related toxicities, while higher dose of radiotherapy on the tumor may be associated with a better survival.

\section{Abbreviations}

$\mathrm{FA}=$ Fanconi anemia, HNSCC = head and neck squamous cell carcinoma, CT = computer tomography, DCE-MRI = dynamic contrast-enhanced magnetic resonance imaging, NCCN = National Comprehensive Cancer Network, IMRT = intensity modulated radiation therapy, RTOG = Radiation Therapy Oncology Group, IFAR = International Fanconi Anemia Registry.

\section{Declarations}

\section{Ethics approval and consent to participate}

This study was in line with the Declaration of Helsinki and was approved by the Ethics Committee of Sichuan Cancer Hospital \& Institute.

\section{Consent for publication}

The patient's parents have provided the written informed consent for this article.

\section{Availability of data and materials}

Not applicable.

\section{Competing interests}


The authors have declared that they have no competing interests in this section.

\section{Funding}

This work was supported by the Department of Science and Technology of Sichuan Province [20YYJC3815, 20GJHZ0088].

\section{Authors' contributions}

Genetic testing: DS and YW; Writing-original draft: DS and SY; Management of the patient: ZS, ZP and ZJ; Resources and Funding acquisition: LJ and LS; Writing-review \& editing: DS and LS. All authors read and approved the final manuscript.

\section{Acknowledgements}

We thank all of our colleagues who have contributed to this article.

\section{References}

1. Bhandari J, Thada PK, Puckett Y. Fanconi Anemia. StatPearls. Treasure Island (FL): StatPearls Publishing Copyright (c) 2020, StatPearls Publishing LLC.; 2020.

2. Furlong E, Carter T. Aplastic anaemia: Current concepts in diagnosis and management. Journal of paediatrics and child health. 2020;56(7):1023-8.

3. Kutler DI, Singh B, Satagopan J, Batish SD, Berwick M, Giampietro PF, et al. A 20-year perspective on the International Fanconi Anemia Registry (IFAR). Blood. 2003;101(4):1249-56.

4. Solomon PJ, Margaret P, Rajendran R, Ramalingam R, Menezes GA, Shirley AS, et al. A case report and literature review of Fanconi Anemia (FA) diagnosed by genetic testing. Italian journal of pediatrics. 2015;41:38.

5. Meetei AR, Levitus M, Xue Y, Medhurst AL, Zwaan M, Ling C, et al. X-linked inheritance of Fanconi anemia complementation group B. Nature genetics. 2004;36(11):1219-24.

6. Ceccaldi R, Sarangi P, D'Andrea AD. The Fanconi anaemia pathway: new players and new functions. Nature reviews Molecular cell biology. 2016;17(6):337-49.

7. Bhattacharjee S, Nandi S. DNA damage response and cancer therapeutics through the lens of the Fanconi Anemia DNA repair pathway. Cell communication and signaling : CCS. 2017;15(1):41.

8. Cheung RS, Taniguchi T. Recent insights into the molecular basis of Fanconi anemia: genes, modifiers, and drivers. International journal of hematology. 2017;106(3):335-44.

9. Sims AE, Spiteri E, Sims RJ, 3rd, Arita AG, Lach FP, Landers T, et al. FANCl is a second monoubiquitinated member of the Fanconi anemia pathway. Nature structural \& molecular biology. 2007;14(6):564-7. 
10. Smogorzewska A, Matsuoka S, Vinciguerra P, McDonald ER, 3rd, Hurov KE, Luo J, et al. Identification of the FANCI protein, a monoubiquitinated FANCD2 paralog required for DNA repair. Cell. 2007;129(2):289-301.

11. D'Andrea AD. Susceptibility pathways in Fanconi's anemia and breast cancer. The New England journal of medicine. 2010;362(20):1909-19.

12. Medhurst AL, Laghmani el H, Steltenpool J, Ferrer M, Fontaine C, de Groot J, et al. Evidence for subcomplexes in the Fanconi anemia pathway. Blood. 2006;108(6):2072-80.

13. Sumpter R, Jr., Levine B. Emerging functions of the Fanconi anemia pathway at a glance. Journal of cell science. 2017;130(16):2657-62.

14. Scheckenbach K, Wagenmann M, Freund M, Schipper J, Hanenberg H. Squamous cell carcinomas of the head and neck in Fanconi anemia: risk, prevention, therapy, and the need for guidelines. Klinische Padiatrie. 2012;224(3):132-8.

15. Kutler DI, Auerbach AD, Satagopan J, Giampietro PF, Batish SD, Huvos AG, et al. High incidence of head and neck squamous cell carcinoma in patients with Fanconi anemia. Arch Otolaryngol Head Neck Surg. 2003;129(1):106-12.

16. Alter BP. Fanconi anemia and the development of leukemia. Best practice \& research Clinical haematology. 2014;27(3-4):214-21.

17. Salum FG, Martins GB, de Figueiredo MA, Cherubini K, Yurgel LS, Torres-Pereira C. Squamous cell carcinoma of the tongue after bone marrow transplantation in a patient with Fanconi anemia. Brazilian dental journal. 2006;17(2):161-5.

18. Guardiola P, Socié G, Li X, Ribaud P, Devergie A, Espérou H, et al. Acute graft-versus-host disease in patients with Fanconi anemia or acquired aplastic anemia undergoing bone marrow transplantation from HLA-identical sibling donors: risk factors and influence on outcome. Blood. 2004;103(1):73-7.

19. Spanier G, Pohl F, Giese T, Meier JK, Koelbl O, Reichert TE. Fatal course of tonsillar squamous cell carcinoma associated with Fanconi anaemia: a mini review. Journal of cranio-maxillo-facial surgery : official publication of the European Association for Cranio-Maxillo-Facial Surgery. 2012;40(6):510-5.

20. Francies FZ, Wainwright R, Poole J, De Leeneer K, Coene I, Wieme G, et al. Diagnosis of Fanconi Anaemia by ionising radiation- or mitomycin C-induced micronuclei. DNA repair. 2018;61:17-24.

21. Rosenberg PS, Tamary H, Alter BP. How high are carrier frequencies of rare recessive syndromes? Contemporary estimates for Fanconi Anemia in the United States and Israel. American journal of medical genetics Part A. 2011;155a(8):1877-83.

22. Kutler DI, Auerbach AD. Fanconi anemia in Ashkenazi Jews. Familial cancer. 2004;3(3-4):241-8.

23. Callén E, Casado JA, Tischkowitz MD, Bueren JA, Creus A, Marcos R, et al. A common founder mutation in FANCA underlies the world's highest prevalence of Fanconi anemia in Gypsy families from Spain. Blood. 2005;105(5):1946-9.

24. Rosenberg PS, Greene MH, Alter BP. Cancer incidence in persons with Fanconi anemia. Blood. 2003;101(3):822-6. 
25. Risitano AM, Marotta S, Calzone R, Grimaldi F, Zatterale A. Twenty years of the Italian Fanconi Anemia Registry: where we stand and what remains to be learned. Haematologica. 2016;101(3):31927.

26. Alter BP, Greene MH, Velazquez I, Rosenberg PS. Cancer in Fanconi anemia. Blood. 2003;101(5):2072.

27. Asur RS, Kimble DC, Lach FP, Jung M, Donovan FX, Kamat A, et al. Somatic mosaicism of an intragenic FANCB duplication in both fibroblast and peripheral blood cells observed in a Fanconi anemia patient leads to milder phenotype. Molecular genetics \& genomic medicine. 2018;6(1):77-91.

28. Fiesco-Roa MO, Giri N, McReynolds LJ, Best AF, Alter BP. Genotype-phenotype associations in Fanconi anemia: A literature review. Blood reviews. 2019;37:100589.

29. Kutler DI, Patel KR, Auerbach AD, Kennedy J, Lach FP, Sanborn E, et al. Natural history and management of Fanconi anemia patients with head and neck cancer: A 10-year follow-up. Laryngoscope. 2016;126(4):870-9.

30. Wong WM, Parvathaneni U, Jewell PD, Martins RG, Futran ND, Laramore GE, et al. Squamous cell carcinoma of the oral tongue in a patient with Fanconi anemia treated with radiotherapy and concurrent cetuximab: a case report and review of the literature. Head \& neck. 2013;35(10):E292-8.

31. Marur S, Forastiere AA. Head and Neck Squamous Cell Carcinoma: Update on Epidemiology, Diagnosis, and Treatment. Mayo Clin Proc. 2016;91(3):386-96.

32. Birkeland AC, Auerbach AD, Sanborn E, Parashar B, Kuhel WI, Chandrasekharappa SC, et al. Postoperative clinical radiosensitivity in patients with fanconi anemia and head and neck squamous cell carcinoma. Archives of otolaryngology-head \& neck surgery. 2011;137(9):930-4.

33. Alter BP. Radiosensitivity in Fanconi's anemia patients. Radiother Oncol. 2002;62(3):345-7.

34. Bremer M, Schindler D, Gross M, Dork T, Morlot S, Karstens JH. Fanconi's anemia and clinical radiosensitivity report on two adult patients with locally advanced solid tumors treated by radiotherapy. Strahlenther Onkol. 2003;179(11):748-53.

35. Budrukkar A, Shahid T, Murthy V, Hussain T, Mulherkar R, Vundinti BR, et al. Squamous cell carcinoma of base of tongue in a patient with Fanconi's anemia treated with radiation therapy: case report and review of literature. Head Neck. 2010;32(10):1422-7.

36. Bernard ME, Kim H, Berhane H, Epperly MW, Franicola D, Zhang X, et al. GS-nitroxide (JP4-039)mediated radioprotection of human Fanconi anemia cell lines. Radiation research. 2011;176(5):60312.

\section{Figures}



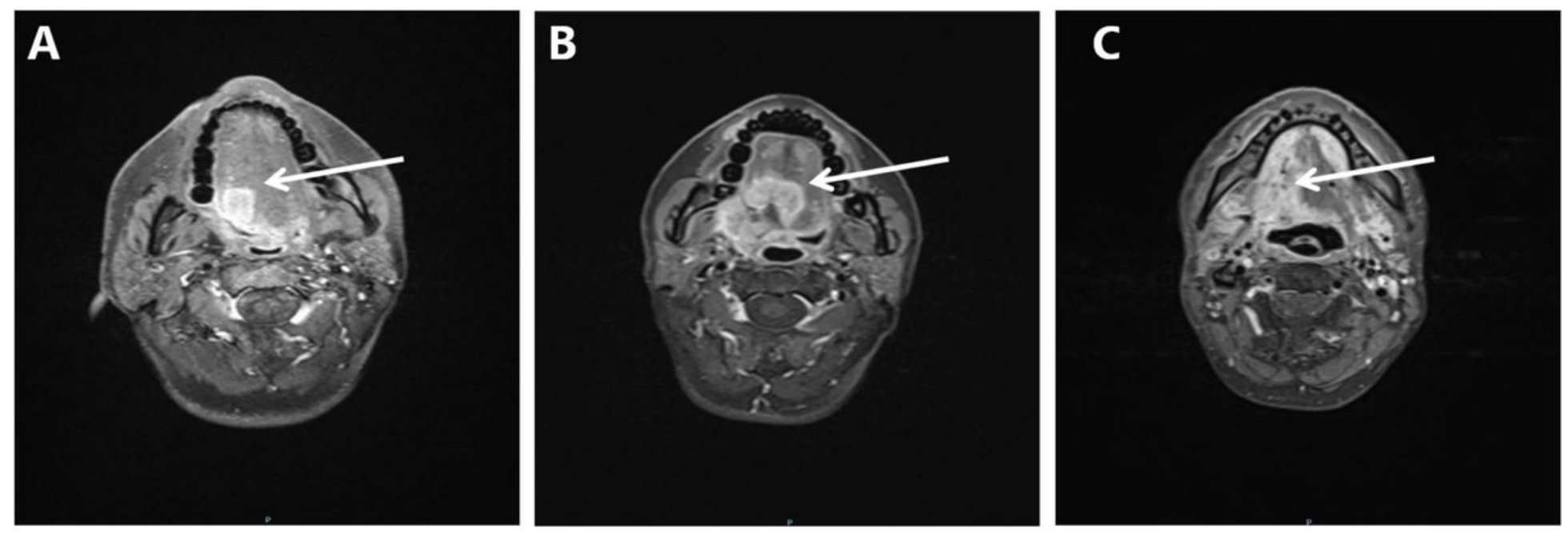

Figure 1

DCE-MRI of the maxillofacial region showed mass located at the right side of tongue dorsum. (A) DCEMRI before the treatment with concurrent cisplatin/radiation, the tumor size measured $1.6 \times 1.4 \times 1.7 \mathrm{~cm}$.

(B) DCE-MRI during the 8 months of bone marrow recovery therapy with cessation of concurrent cisplatin/radiation, the tumor size had increased to $3.7 \times 3.0 \times 3.1 \mathrm{~cm}$. (C) DCE-MRI after completion of 21 fractions of reduced-dose radiotherapy, the tumor size had decreased to $3.2 \times 2.3 \times 2.8 \mathrm{~cm}$. DCE-MRI, dynamic contrast-enhanced magnetic resonance imaging.
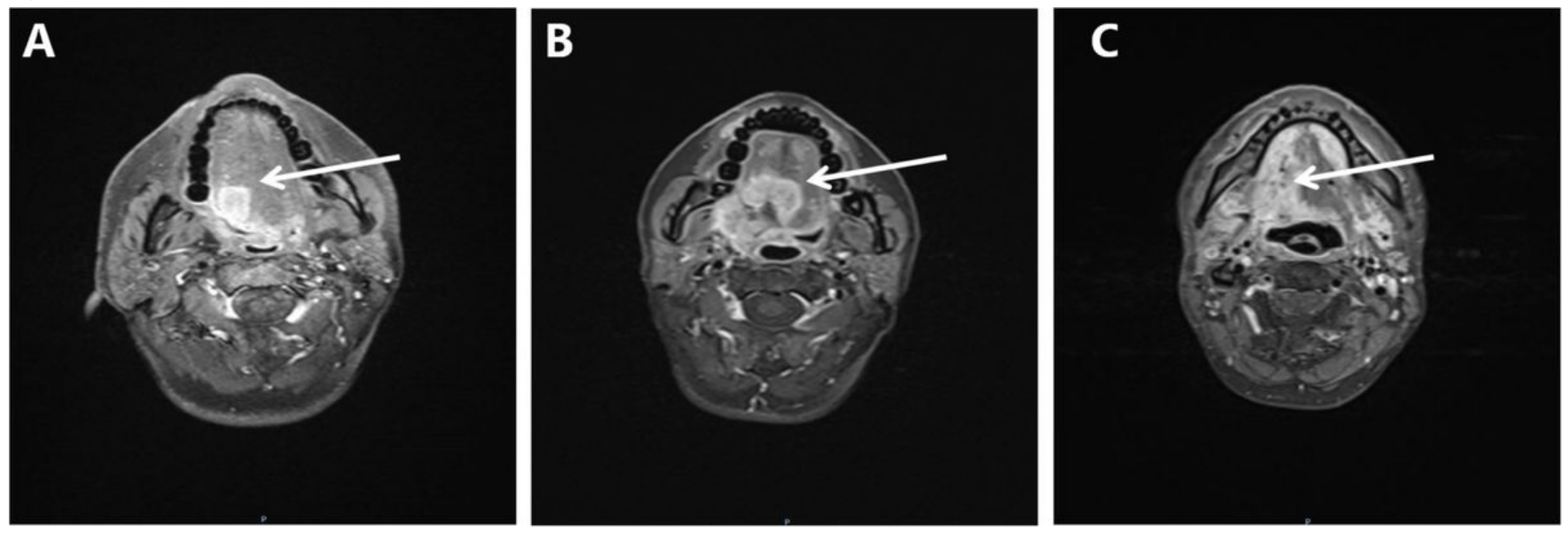

\section{Figure 1}

DCE-MRI of the maxillofacial region showed mass located at the right side of tongue dorsum. (A) DCEMRI before the treatment with concurrent cisplatin/radiation, the tumor size measured $1.6 \times 1.4 \times 1.7 \mathrm{~cm}$. (B) DCE-MRI during the 8 months of bone marrow recovery therapy with cessation of concurrent cisplatin/radiation, the tumor size had increased to $3.7 \times 3.0 \times 3.1 \mathrm{~cm}$. (C) DCE-MRI after completion of 21 fractions of reduced-dose radiotherapy, the tumor size had decreased to $3.2 \times 2.3 \times 2.8 \mathrm{~cm}$. DCE-MRI, dynamic contrast-enhanced magnetic resonance imaging. 

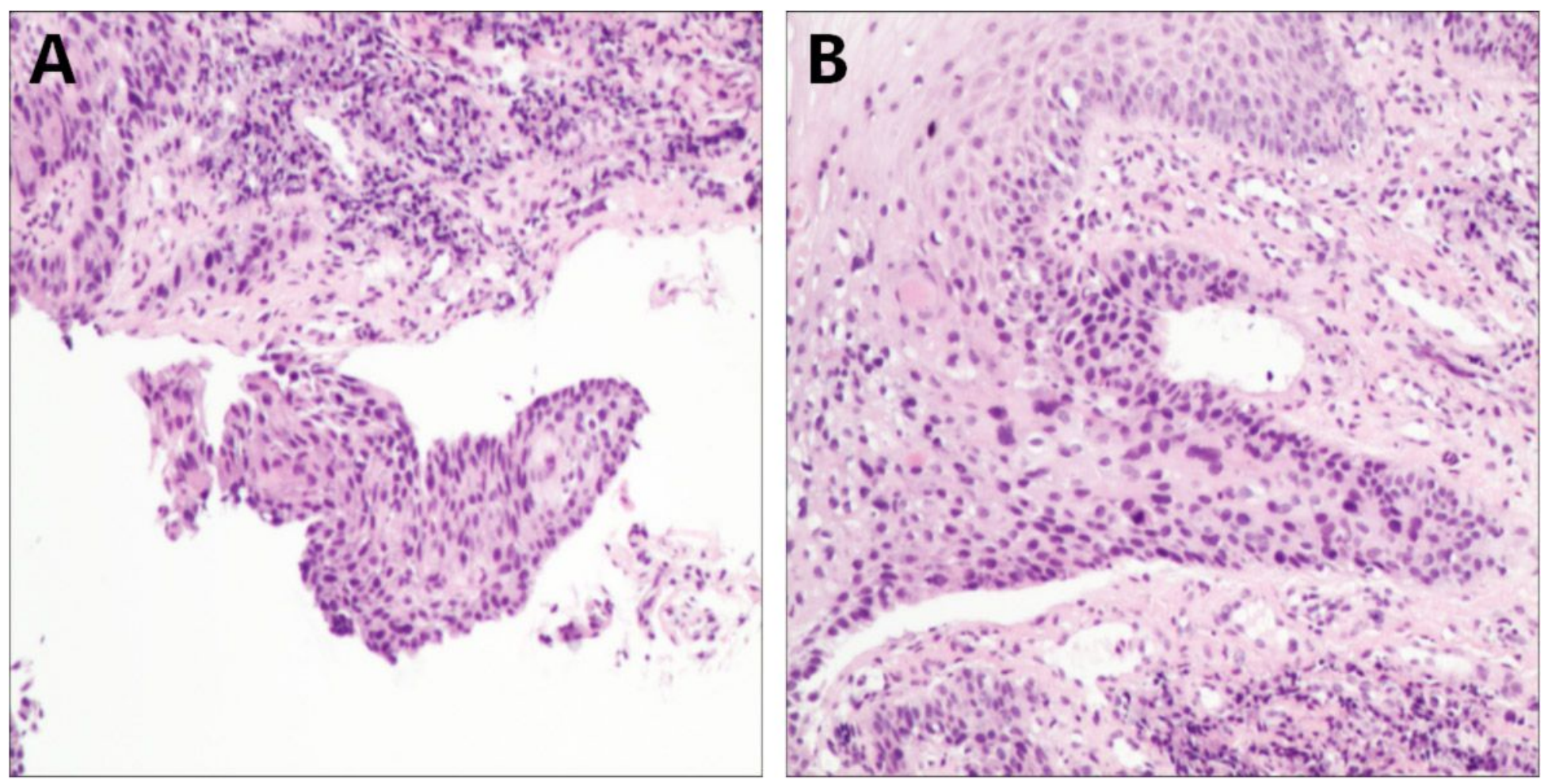

Figure 2

A pathological biopsy demonstrated highly differentiated squamous cell carcinoma. (A) Hematoxylineosin, original magnification 40X. (B) Hematoxylin-eosin, original magnification 200X.
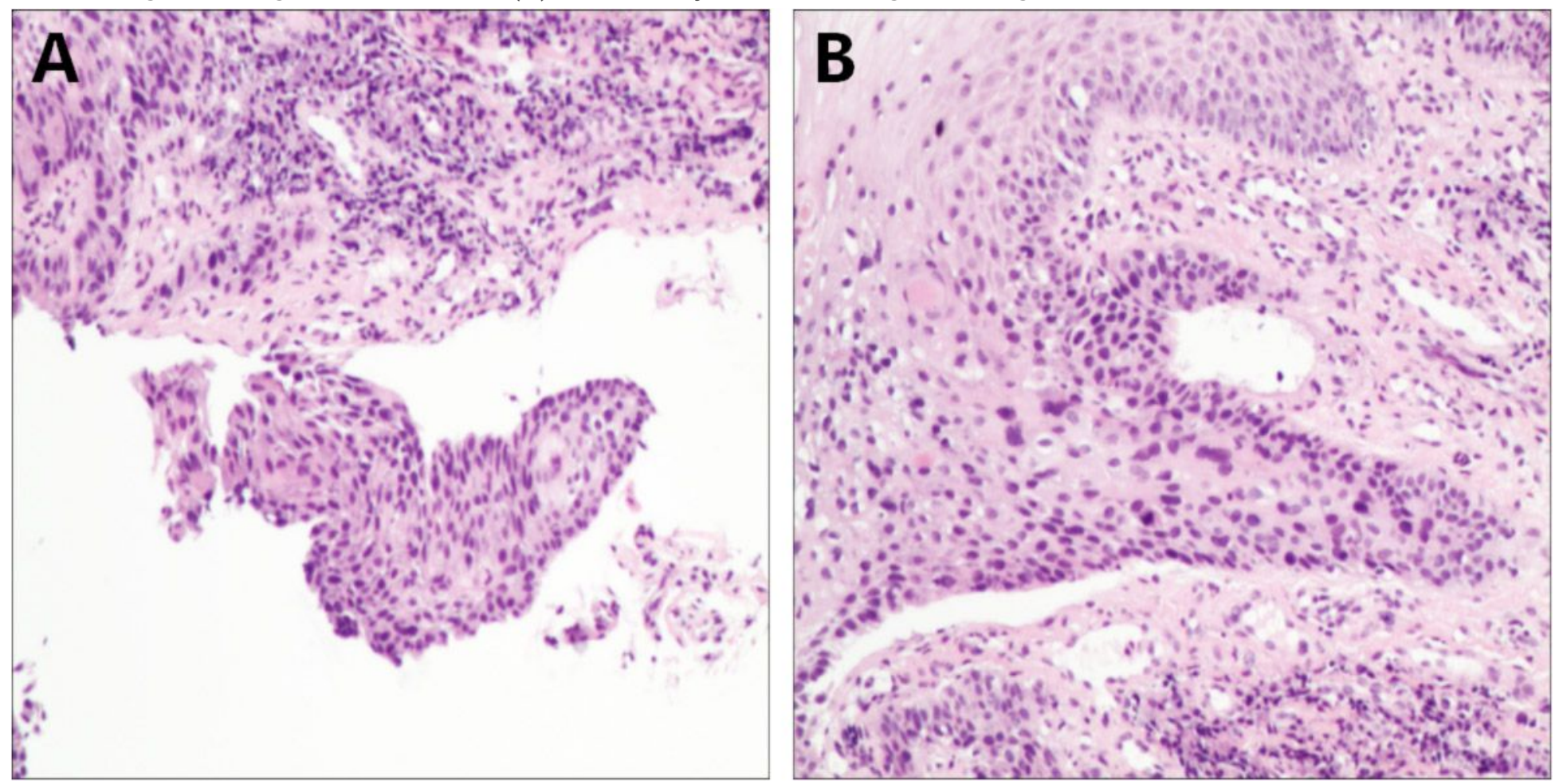

Figure 2 
A pathological biopsy demonstrated highly differentiated squamous cell carcinoma. (A) Hematoxylineosin, original magnification 40X. (B) Hematoxylin-eosin, original magnification 200X.
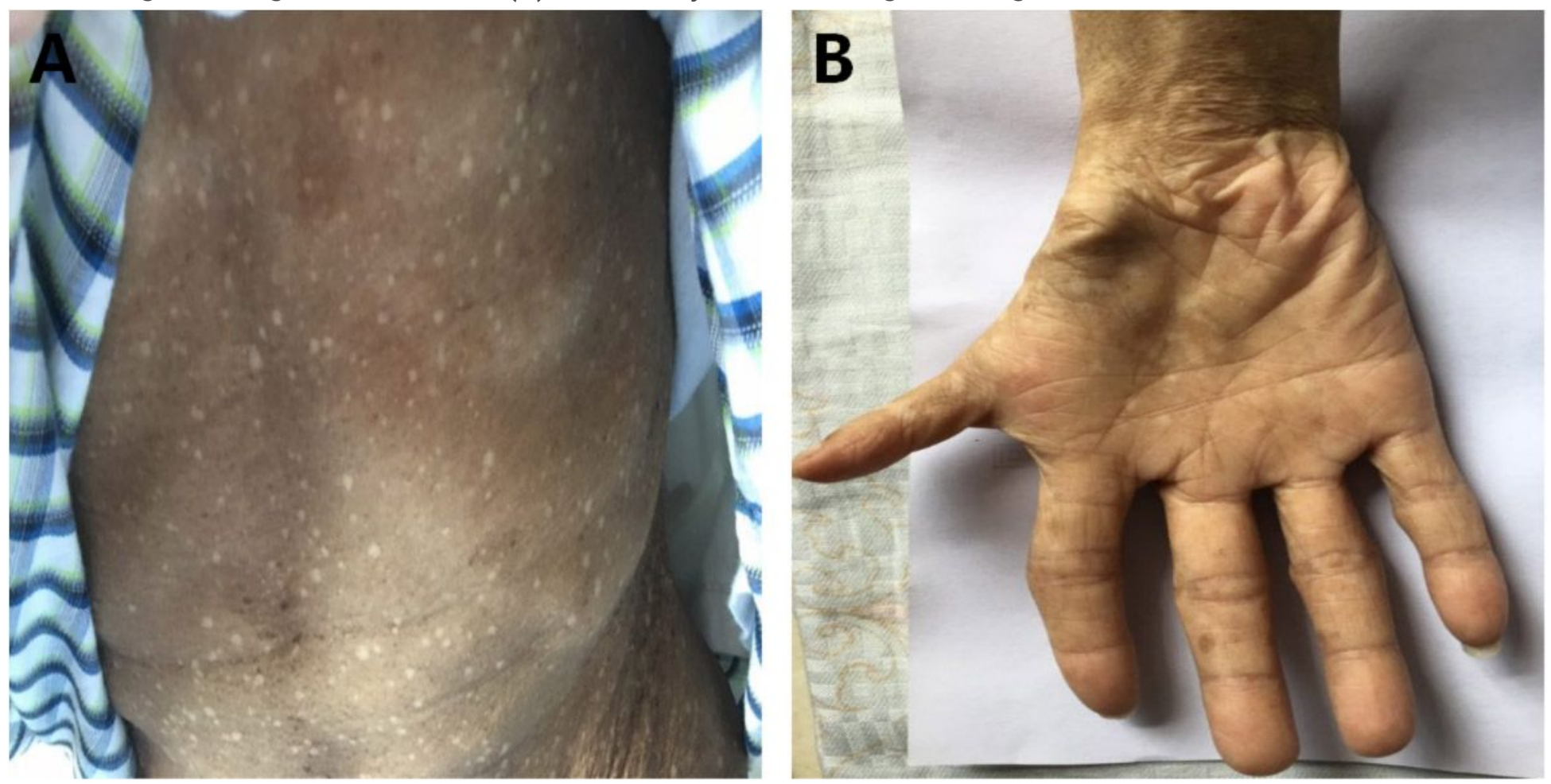

\section{Figure 3}

(A) and (B). Physical examination showed generalized hyperpigmentation of the skin, accompanied by a few café au lait spots, particularly of the upper extremities.
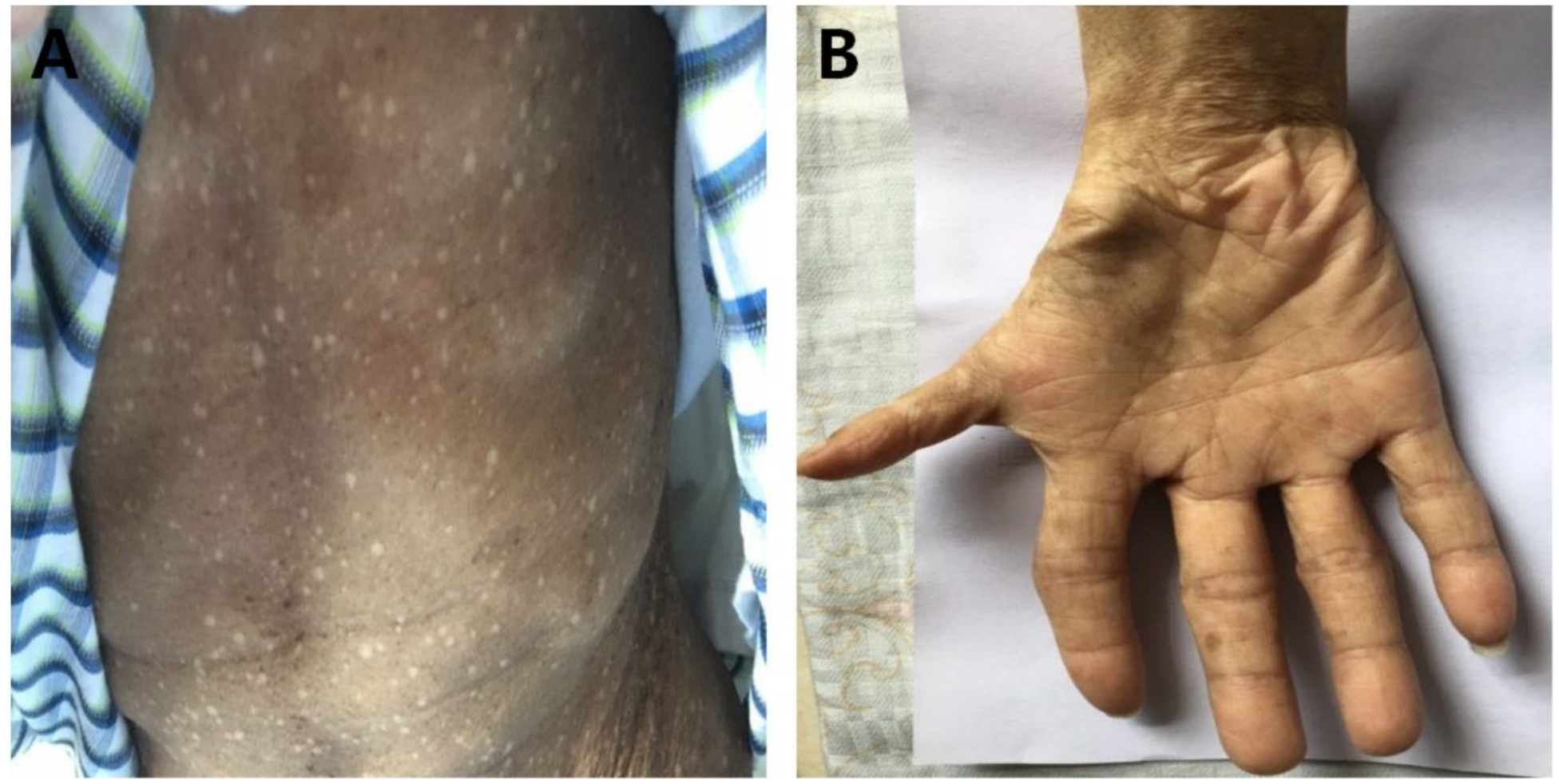

Figure 3 
(A) and (B). Physical examination showed generalized hyperpigmentation of the skin, accompanied by a few café au lait spots, particularly of the upper extremities.

A Reference genome sequence C CT CTAG C G T G G G A C A G A T C T G C A C G G C T C

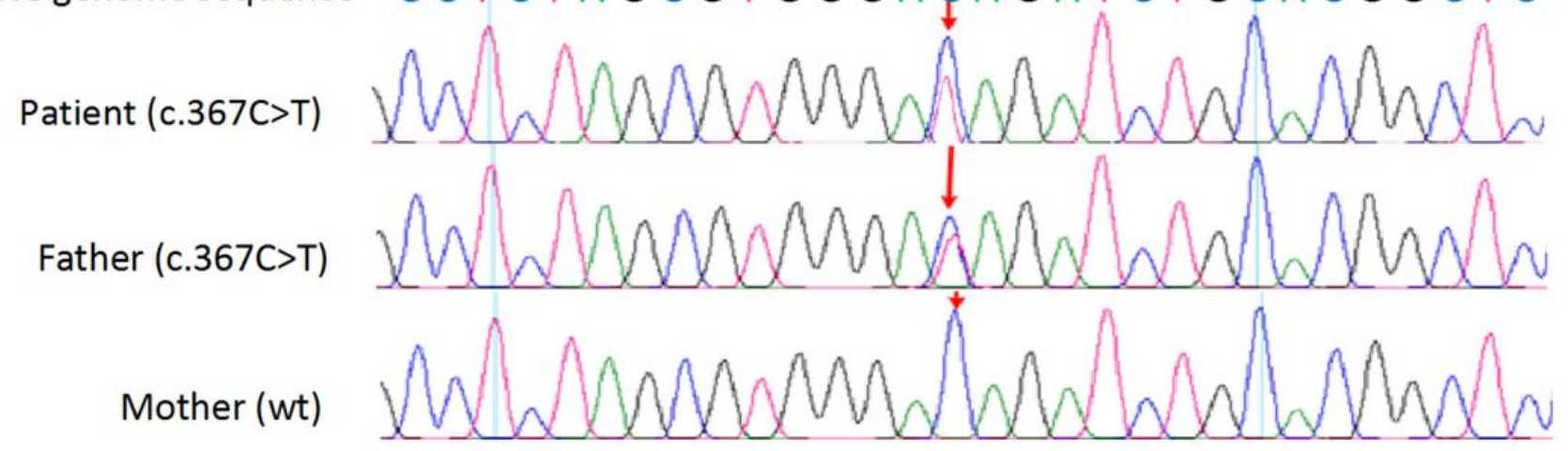

B Reference genome sequence C CT C C GTGTG G C C C CGGATCAG CA C G C CAGG C Patient (c.3971_3972delCGinsTT)

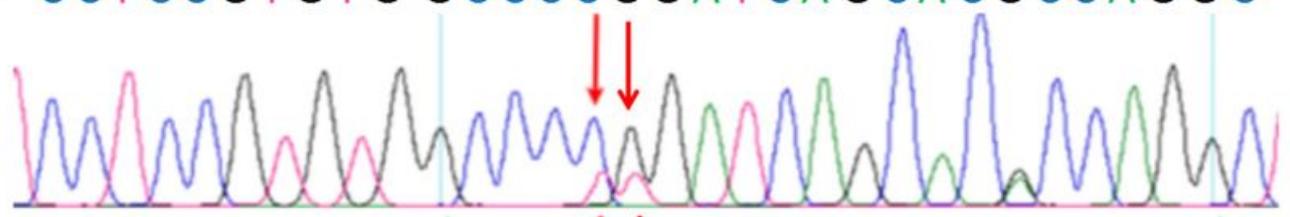

Father (wt)

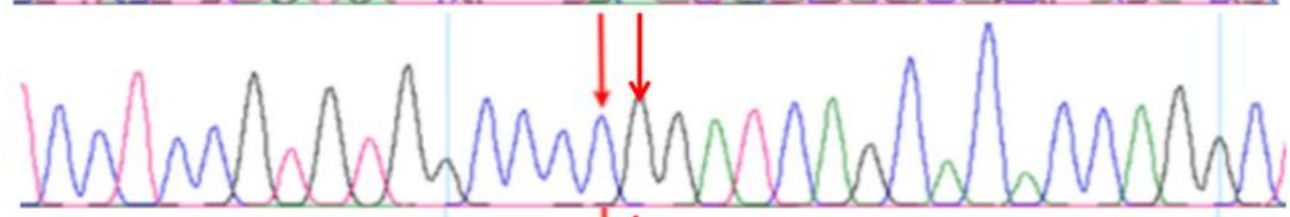

Mother (c.3971_3972delcGinstT) $\mathrm{Am} A \mathrm{Al}$

\section{Figure 4}

Fanconi anemia complementation group A gene sequence diagram of the patient. (A) c.367C>T was detected in the patient and his father. (B) c.3971_3972delCGinsTT was detected in the patient and his mother. Variants were indicated with arrows; wt, wild type. 
A Reference genome sequence C C T C TA G C G T G G G A C A G A T C T G C A C G G C T C

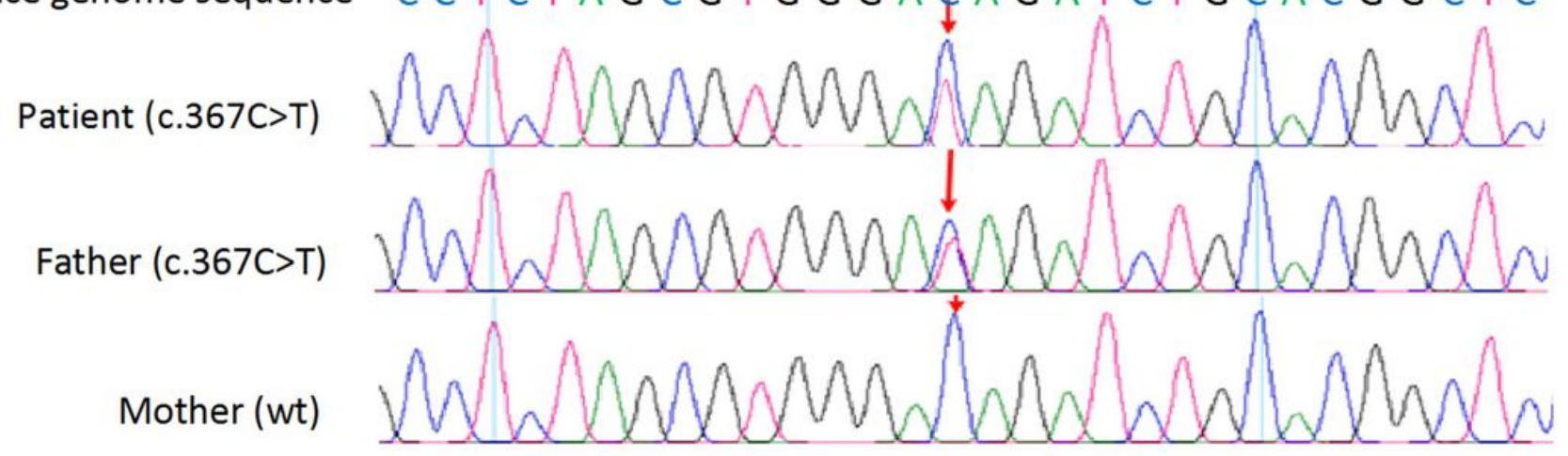

B Reference genome sequence C CT C C GTG TG G C C C C G G AT CA G CA C G C CAGG C Patient (c.3971_3972delCGinsTT)

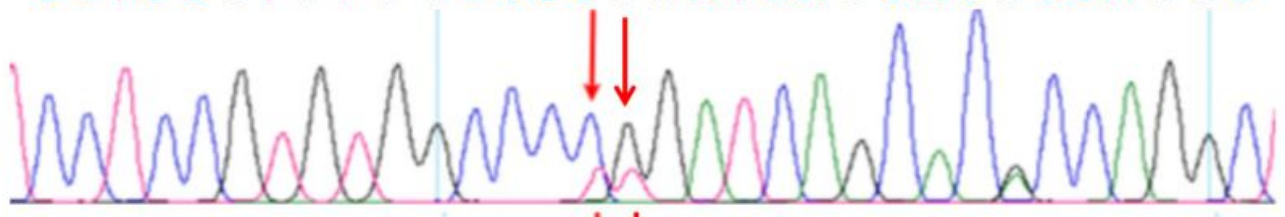

Father (wt)

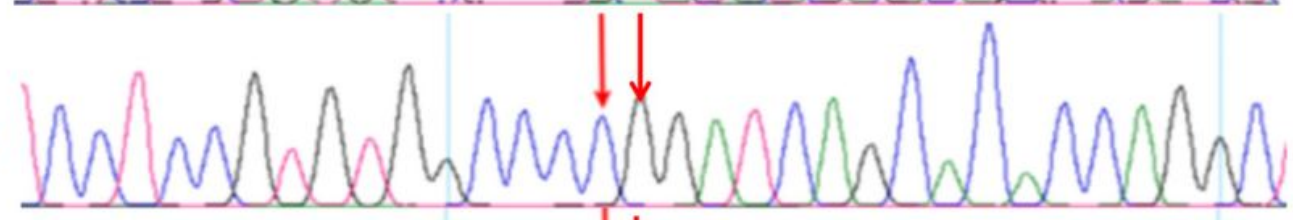

Mother (c.3971_3972delCGinstT) $\mathrm{Am}$

\section{Figure 4}

Fanconi anemia complementation group A gene sequence diagram of the patient. (A) c.367C >T was detected in the patient and his father. (B) c.3971_3972delCGinsTT was detected in the patient and his mother. Variants were indicated with arrows; wt, wild type. 


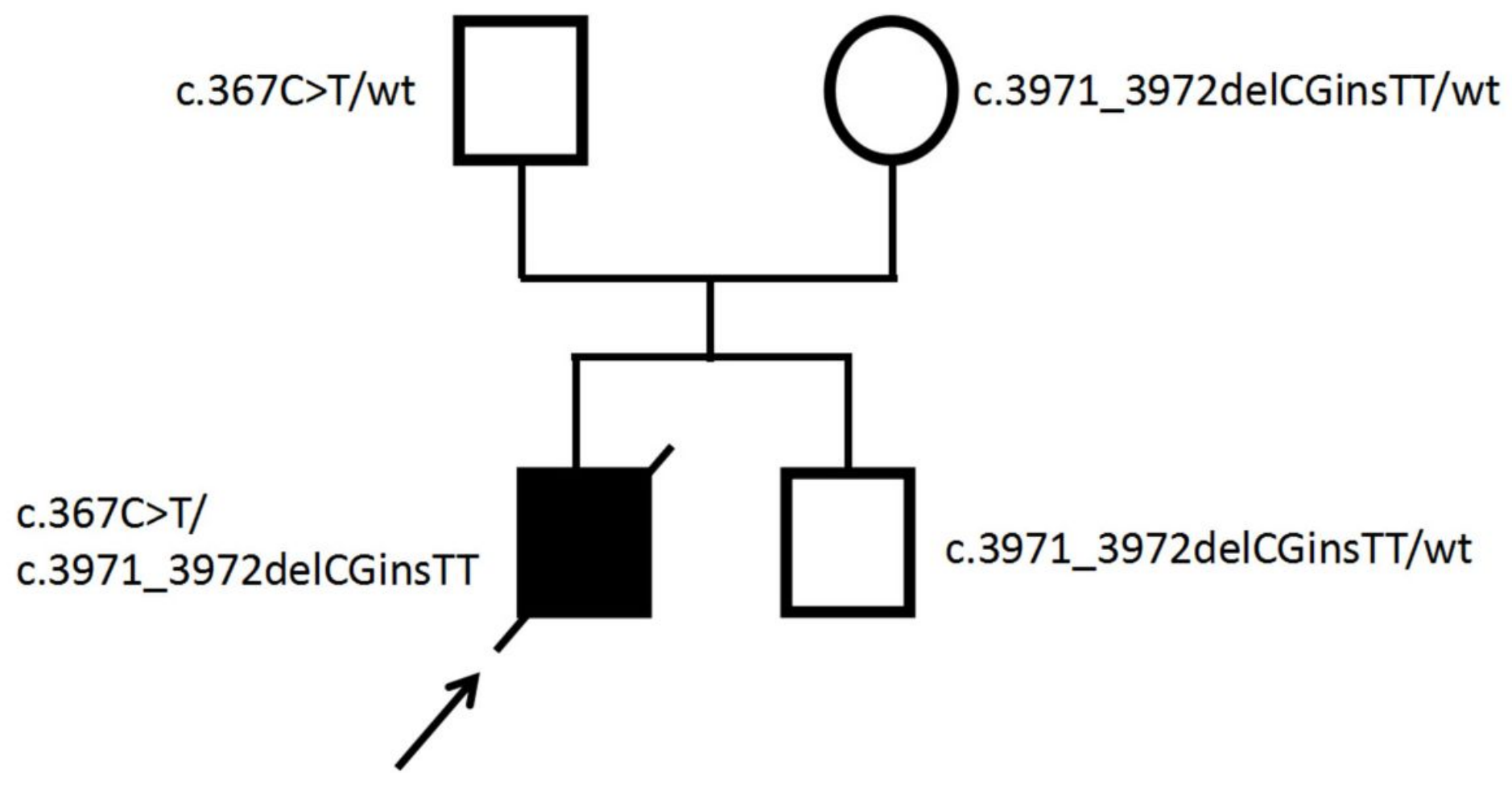

Figure 5

Pedigree of the family. Filled and open symbols denoted affected and healthy individuals, respectively. An arrow indicated the index patient; diagonal line indicated deceased status. The mutation status was shown next to each symbol; wt, wild type.

c. $367 \mathrm{C}>\mathrm{T} /$

c.3971_3972delCGinsTT

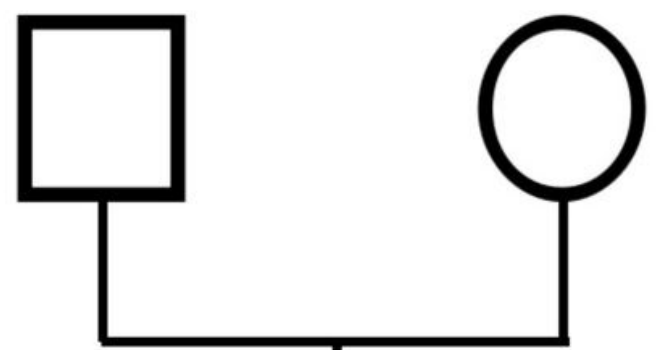

c.3971_3972delCGinsTT/wt

c.3971_3972delCGinsTT/wt

\section{Figure 5}


Pedigree of the family. Filled and open symbols denoted affected and healthy individuals, respectively. An arrow indicated the index patient; diagonal line indicated deceased status. The mutation status was shown next to each symbol; wt, wild type. 